Journal of Applied Analysis

Vol. 14, No. 2 (2008), pp. 239-249

\title{
BOUNDED CONTROLLERS FOR UNCERTAIN NONLINEAR SYSTEMS
}

\author{
A. BENABDALLAH and M. A. HAMMAMI
}

Received October 17, 2006 and, in revised form, July 1, 2008

\begin{abstract}
In this paper, we study the stabilization problem of uncertain systems. We treat a class of uncertain systems whose nominal part is affine in the control and whose uncertain part is bounded by a known affine function of the control, when the control is bounded by a specified constant.
\end{abstract}

\section{INTRODUCTION}

Dynamical systems with uncertainties have attracted considerable attention in control literature, particularly uncertain systems with linear nominal part (system without uncertainties). For uncertain systems with linear nominal part, the problem of state observation was considered in [5] and [14], sufficient conditions for the existence of an output feedback stabilizing controller were given in [1] and [5], stabilizing bounded controllers were proposed in [2], [3], [4], [6], [7], [10], [11], [13].

The stabilization of affine in the control systems has been widely investigated in recent years because of their capability of modelling a large number

2000 Mathematics Subject Classification. Primary: 34D40, 34D23, 34D20, 34D10.

Key words and phrases. Uncertain nonlinear systems, bounded controllers, stabilization.

ISSN 1425-6908（C) Heldermann Verlag. 
of processes and their intrinsic simplicity. The present work considers the stabilization of nonlinear uncertain systems whose nominal part is affine. It extends the result of Corless and Leitmann [4]. In [4], Corless and Leitmann have addressed the stabilization of nonlinear uncertain systems whose nominal part is linear. Subject to a controller prescribed constraint, they have proposed controllers that guarantee the uniform exponential convergence of the solutions towards a neighborhood of the origin. The proposed controller depends on the solution to the Riccati equation. From optimal control theory, we see that the Riccati equation is used to derive the optimal state feedback control law for the linear system with a quadratic cost functional. It is well known $([9])$ that for affine in the control systems with arbitrary cost functional an optimal state feedback control law can be derived from the solution to the Hamilton-Jacobi-Bellman equation. This motivated us to consider an uncertain system with affine nominal part.

In this paper, we will consider an uncertain system with affine nominal part. Subject to a controller constraint, we will give sufficient conditions for designing a controller that guarantees the uniform exponential convergence of the solutions towards an arbitrary small neighborhood of the origin. In [15], Wu and Mizukami investigated the stabilization of such a class of systems, but there was no given controller constraint. Our work is organized as follows. In Section 2, we recall the definition of uniform exponential convergence to a neighborhood of the origin. We give also a sufficient condition for uniform exponential convergence. In Section 3, we present bounded controllers that guarantee uniform exponential convergence of solutions of the considered system to a neighborhood of the origin with a specified rate of convergence.

\section{Mathematical PREliminaries}

Consider a system described by

$$
\dot{x}=F(t, x)
$$

where $t \in \mathbb{R}_{+}$is the time, $x \in \mathbb{R}^{n}$ is the state, $F: \mathbb{R}_{+} \times \mathbb{R}^{n} \rightarrow \mathbb{R}^{n}$ is continuous in $t$ locally Lipschitz in $x$ uniformly in $t$. Let $\alpha>0$ and $r \geq 0$ and define

$$
B(r)=\left\{x \in \mathbb{R}^{n} \mid\|x\| \leq r\right\} .
$$

Suppose that $R \subset \mathbb{R}^{n}$. We first give the definition of uniform exponential convergence of (1) towards $B(r)$ with rate $\alpha$ and region of attraction $R$.

Definition 1. System (1) is uniformly exponentially convergent to $B(r)$ with rate $\alpha>0$ and region of attraction $R$, if there exists a real scalar 
$\beta \geq 0$ such that, if

$$
x:\left[t_{0}, \infty\right) \rightarrow \mathbb{R}^{n}
$$

is any solution of $(1)$ with $x\left(t_{0}\right) \in R$, then

$$
\|x(t)\| \leq r+\beta\left\|x\left(t_{0}\right)\right\| \exp \left[-\alpha\left(t-t_{0}\right)\right], \quad \forall t \geq t_{0} .
$$

Definition 2. System (1) is globally uniformly exponentially convergent to $B(r)$ if it is uniformly exponentially convergent with $\mathbb{R}^{n}$ as a region of attraction.

We recall now a sufficient condition to assure uniform exponential convergence.

Theorem 1 ([4]). Consider system (1). Suppose that there exist a $C^{1}$ function $V: \mathbb{R}^{n} \rightarrow \mathbb{R}$ and real numbers $\alpha, \lambda_{1}, \lambda_{2}, V_{1}, V_{2}$, with

$$
0<\alpha, \lambda_{1}, \lambda_{2}<\infty
$$

and

$$
0 \leq V_{1}<V_{2} \leq \infty
$$

such that the following inequalities hold for all $t \in \mathbb{R}_{+}$

$$
\lambda_{1}\|x\|^{2} \leq V(x) \leq \lambda_{2}\|x\|^{2}
$$

for all $x \in \mathbb{R}^{n}$ and

$$
D V(x) F(t, x) \leq-2 \alpha\left(V(x)-V_{1}\right)
$$

for all $x$ which satisfy $V_{1}<V(x)<V_{2}$. Then, letting

$$
r:=\left(\frac{V_{1}}{\lambda_{1}}\right)^{1 / 2},
$$

system (1) is uniformly exponentially convergent to $B(r)$ with rate $\alpha$ and region of attraction

$$
R=\left\{x \in \mathbb{R}^{n} \mid V(x) \leq V_{2}\right\} .
$$

\section{Main Result}

Throughout this paper, we deal with uncertain dynamical systems described by

$$
\dot{x}=f(x)+g(x) u+E(t, x, u)
$$

where $t \in \mathbb{R}_{+}$is the time, $x(t) \in \mathbb{R}^{n}$ is the state, $u(t) \in \mathbb{R}^{m}$ is the control input and $f: \mathbb{R}^{n} \rightarrow \mathbb{R}^{n}, g: \mathbb{R}^{n} \rightarrow \mathbb{R}^{n \times m}$ are known functions. The function 
$E: \mathbb{R}_{+} \times \mathbb{R}^{n} \times \mathbb{R}^{m} \rightarrow \mathbb{R}^{n}$ represents uncertainties in the plant. The nominal system corresponding to system (2) is given by

$$
\dot{x}=f(x)+g(x) u \text {. }
$$

The control $u$ is subject to the constraint

$$
\|u\| \leq \bar{\rho}
$$

where $\bar{\rho}$ is prescribed. Our aim is to design a state controller satisfying (4) such that system (2) is uniformly convergent towards a small neighborhood of the origin. We consider the following assumptions pertaining to system (2).

$\left(A_{1}\right)$ There exists a function $h$ such that

$$
E(t, x, u)=g(x) h(t, x, u) .
$$

$\left(A_{2}\right)$ There exist nonnegative real scalars $k_{1}, k_{2}$, with $k_{2}<1$, such that

$$
\|h(t, x, u)\| \leq k_{1}+k_{2}\|u\|
$$

for all $t \in \mathbb{R}_{+}, x \in \mathbb{R}^{n}, u \in \mathbb{R}^{m}$.

$\left(A_{3}\right)$ The numbers $k_{1}$ and $k_{2}$ satisfy

$$
\frac{k_{1}}{1-k_{2}}<\bar{\rho}
$$

We will consider the problem of choosing $u$ subject to the controller constraint (4) such that, for all uncertainties satisfying $\left(A_{1}\right),\left(A_{2}\right)$ and $\left(A_{3}\right)$, system (2) is uniformly convergent to an arbitrary small neighborhood of the origin. It is worth noting that in literature assumption $\left(A_{1}\right)$ is referred to as the "matching condition".

\subsection{Unconstrained controllers.}

Let $\alpha>0$. We will consider, in this section, the problem of choosing $u$ so that, for all uncertainties satisfying $\left(A_{1}\right)$ and $\left(A_{2}\right)$, system $(2)$ is globally uniformly exponentially convergent to a given ball $B(r)$ with rate $\alpha$. We suppose that the assumption below is fulfilled.

$\left(A_{4}\right)$ There exists a $C^{1}$ function $V: \mathbb{R}^{n} \rightarrow \mathbb{R}$ which satisfies

$$
2 \alpha V(x)+L_{f} V(x)-L_{g} V(x)\left(L_{g} V(x)\right)^{T} \leq 0
$$

where $L_{f} V$ denotes the Lie derivative of $V$ along $f$. Moreover, there exist positive constants $\lambda_{1}, \lambda_{2}$ and $\lambda_{3}$ such that

$$
\begin{gathered}
\lambda_{1}\|x\|^{2} \leq V(x) \leq \lambda_{2}\|x\|^{2}, \\
\|D V(x)\| \leq \lambda_{3}\|x\|
\end{gathered}
$$

for all $x \in \mathbb{R}^{n}$. 
For any $\varepsilon>0$, the proposed controller is given by

$$
u(x)=-\left(1-k_{2}\right)^{-1}\left(L_{g} V(x)\right)^{T}-\rho s\left(\varepsilon^{-1}\left(L_{g} V(x)\right)^{T}\right)
$$

where $V$ is the Lyapunov function given by assumption $\left(A_{4}\right)$,

$$
\rho=\left(1-k_{2}\right)^{-1} k_{1}
$$

and the function $s$ is given by

$$
s(y)=(1+\|y\|)^{-1} y .
$$

We have the following result.

Theorem 2. Consider an uncertain system described by (2) satisfying assumptions $\left(A_{1}\right),\left(A_{2}\right),\left(A_{3}\right)$ and $\left(A_{4}\right)$, and subject to the control given by $(8)$. Then the resulting closed loop system is globally exponentially convergent to $B\left(r_{\varepsilon}\right)$ with rate $\alpha$ where

$$
r_{\varepsilon}=\left(\frac{\varepsilon k_{1}}{2 \alpha \lambda_{1}}\right)^{1 / 2} .
$$

Proof. We will use the function $V$ as a Lyapunov function candidate for the closed loop system. Its derivative along the trajectories of (2) is given by

$$
\begin{aligned}
\dot{V}(t) & =D V(x)(f(x)+g(x) u+E(t, x, u)) \\
& =L_{f} V(x)+L_{g} V(x) u+D V(x) E(t, x, u) .
\end{aligned}
$$

Taking into account assumptions $\left(A_{1}\right)$ and $\left(A_{2}\right)$ we have

$$
\begin{aligned}
\dot{V}(t) & =L_{f} V(x)+L_{g} V(x) u+L_{g} V(x) h(t, x, u) \\
& \leq L_{f} V(x)+L_{g} V(x) u+\left\|L_{g} V(x)\right\|\|h(t, x, u)\| \\
& \leq L_{f} V(x)+L_{g} V(x) u+\left\|L_{g} V(x)\right\|\left(k_{1}+k_{2}\|u\|\right) .
\end{aligned}
$$

Using (8) it follows that

$$
\begin{aligned}
\dot{V}(t) \leq & L_{f} V(x)-\left(1-k_{2}\right)^{-1}\left\|L_{g} V(x)\right\|^{2} \\
& -\rho L_{g} V(x) s\left(\varepsilon^{-1}\left(L_{g} V(x)\right)^{T}\right)+k_{1}\left\|L_{g} V(x)\right\| \\
& +k_{2}\left\|L_{g} V(x)\right\|\left(\left(1-k_{2}\right)^{-1}\left\|L_{g} V(x)\right\|+\rho\left\|s\left(\varepsilon^{-1}\left(L_{g} V(x)\right)^{T}\right)\right\|\right) \\
\leq & L_{f} V(x)-\left(1-k_{2}\right)^{-1}\left\|L_{g} V(x)\right\|^{2}-\rho \frac{\varepsilon^{-1}\left\|L_{g} V(x)\right\|^{2}}{1+\varepsilon^{-1}\left\|L_{g} V(x)\right\|} \\
& +k_{1}\left\|L_{g} V(x)\right\|+k_{2}\left(1-k_{2}\right)^{-1}\left\|L_{g} V(x)\right\|^{2}+k_{2} \rho \frac{\varepsilon^{-1}\left\|L_{g} V(x)\right\|^{2}}{1+\varepsilon^{-1}\left\|L_{g} V(x)\right\|} \\
\leq & L_{f} V(x)-\left\|L_{g} V(x)\right\|^{2}+k_{1}\left\|L_{g} V(x)\right\|-\left(1-k_{2}\right) \rho \frac{\varepsilon^{-1}\left\|L_{g} V(x)\right\|^{2}}{1+\varepsilon^{-1}\left\|L_{g} V(x)\right\|}
\end{aligned}
$$




$$
\begin{aligned}
& =L_{f} V(x)-\left\|L_{g} V(x)\right\|^{2}+k_{1}\left\|L_{g} V(x)\right\|-k_{1} \frac{\varepsilon^{-1}\left\|L_{g} V(x)\right\|^{2}}{1+\varepsilon^{-1}\left\|L_{g} V(x)\right\|} \\
& =L_{f} V(x)-\left\|L_{g} V(x)\right\|^{2}+\frac{k_{1}\left\|L_{g} V(x)\right\|}{1+\varepsilon^{-1}\left\|L_{g} V(x)\right\|} \\
& \leq L_{f} V(x)-\left\|L_{g} V(x)\right\|^{2}+k_{1} \varepsilon .
\end{aligned}
$$

Now, using (5), we have

$$
L_{f} V(x) \leq-2 \alpha V(x)+\left\|L_{g} V(x)\right\|^{2} .
$$

So, we obtain the following upper bound on $\dot{V}$

$$
\dot{V} \leq-2 \alpha\left(V-\frac{k_{1} \varepsilon}{2 \alpha}\right)
$$

Moreover $V$ satisfies (6), so we can use Theorem 1, with

$$
V_{1}=\frac{k_{1} \varepsilon}{2 \alpha}
$$

to conclude.

Remark 1. As in [1]-[5], [13], [15], the controller (8) consists of two parts. The first one,

$$
u_{1}(x)=-\left(L_{g} V(x)\right)^{T},
$$

stabilizes the nominal system and the second one,

$$
u_{2}(x)=-k_{2}\left(1-k_{2}\right)^{-1}\left(L_{g} V(x)\right)^{T}-\rho s\left(\varepsilon^{-1}\left(L_{g} V(x)\right)^{T}\right),
$$

is used to compensate for the system uncertainties and render the uncertain system globally uniformly exponentially convergent to the ball $B\left(r_{\varepsilon}\right)$.

Remark 2. In [4], the nominal system is linear and it is supposed to be stabilized by a linear feedback of the states, where the control gains are obtained by solving a Riccati equation. For system (3), the optimal control is given by (see [9], [12]):

$$
u^{*}(x)=-\frac{1}{2} R^{-1}(x) L_{g} V^{*}(x)^{T}
$$

where $V$ is the solution to the Hamilton-Jacobi-Bellman equation:

$$
L_{f} V^{*}(x)+l(x)-\frac{1}{4} L_{g} V^{*}(x) R^{-1}(x)\left(L_{g} V^{*}(x)\right)^{T}=0, \quad V^{*}(0)=0
$$

where $l(x) \geq 0$ and $R(x)>0$ for all $x$. If there exists a $C^{1}$-function $V^{*}$ that satisfies (6) and that is a solution to (9) for $R>0$ and $l(x)=8 \alpha \lambda_{2} R\|x\|^{2}$, then $V=(1 / 4) R^{-1} V^{*}$ or $V=(1 / 2)\left(R^{-1}+1\right) V^{*}$ (as in [4] for the linear case) satisfies (5). 


\subsection{Constrained controllers.}

It is clear that, controller (8) does not satisfy (4). In this subsection, We will consider the problem of choosing $u$ subject to the constraint (4) such that, there is a region of attraction $R$ from which all solutions of (2) are uniformly exponentially convergent to a given ball $B(r)$ with rate $\alpha$. We suppose that system (2) satisfies the following assumption.

$\left(A_{5}\right) g$ is a globally Lipschitz function with a Lipschitz constant $L$.

The proposed controllers are given by

$$
\bar{u}(x)=-\tilde{\rho} \operatorname{sat}\left(\tilde{\rho}^{-1}\left(1-k_{2}\right)^{-1}\left(L_{g} V(x)\right)^{T}\right)-\rho s\left(\varepsilon^{-1}\left(L_{g} V(x)\right)^{T}\right)
$$

where the saturation function is given by

$$
\operatorname{sat}(y)= \begin{cases}y & \text { if }\|y\| \leq 1 \\ \|y\|^{-1} y & \text { if }\|y\|>1\end{cases}
$$

$\varepsilon$ is any positive real scalar which satisfies

$$
\varepsilon<\frac{\alpha\left(1-k_{2}\right) \tilde{\rho} \lambda_{1}}{k_{1} \lambda_{3} L}
$$

and $\tilde{\rho}=\bar{\rho}-\rho$.

Now we can state the following result.

Theorem 3. Under assumptions $\left(A_{1}\right),\left(A_{2}\right),\left(A_{3}\right),\left(A_{4}\right)$ and $\left(A_{5}\right)$ the closed loop system (2)-(10) is uniformly exponentially convergent to $B\left(r_{\varepsilon}\right)$ with rate $\alpha$ and region of attraction $R$ where

$$
R=\left\{x \in \mathbb{R}^{n} \mid V(x) \leq \frac{\left(1-k_{2}\right) \tilde{\rho} \lambda_{1}}{L \lambda_{3}}\right\} .
$$

Proof. It is clear that if

$$
\left\|L_{g} V(x)\right\| \leq\left(1-k_{2}\right) \tilde{\rho}
$$

we have

$$
\bar{u}(x)=u(x) .
$$

Moreover, using assumption $\left(A_{5}\right)$ and equation (7), we obtain

$$
\begin{aligned}
\left\|L_{g} V(x)\right\| & \leq\|D V(x)\|\|g(x)\| \\
& \leq \lambda_{3} L\|x\|^{2} \\
& \leq \frac{\lambda_{3} L}{\lambda_{1}} V(x) .
\end{aligned}
$$

So, whenever

$$
V(x) \leq \frac{\left(1-k_{2}\right) \tilde{\rho} \lambda_{1}}{L \lambda_{3}}
$$


we have

$$
\left\|L_{g} V(x)\right\| \leq\left(1-k_{2}\right) \tilde{\rho}
$$

and thus

$$
\bar{u}(x)=u(x) .
$$

We can now proceed as in the proof of Theorem 2. We show that the hypotheses of Theorem 1 are satisfied with

and

$$
V_{1}=\frac{k_{1} \varepsilon}{\alpha}
$$

$$
V_{2}=\frac{\left(1-k_{2}\right) \tilde{\rho} \lambda_{1}}{\lambda_{2} L}
$$

It is worth noting that if we consider as in [4] a class of uncertain systems with linear nominal part, assumption $\left(A_{5}\right)$ is not satisfied. However, such a class of systems will satisfy the assumption below. $\left(A_{5}^{\prime}\right) g$ is globally bounded by a positive constant $M$.

We consider controller (10) where $\varepsilon$ is any positive real scalar which satisfies

$$
\varepsilon<\frac{\alpha\left(1-k_{2}\right)^{2} \tilde{\rho}^{2} \lambda_{1}}{k_{1} \lambda_{3}^{2} M^{2}} .
$$

We may also state the following result.

Theorem 4. Under assumptions $\left(A_{1}\right),\left(A_{2}\right),\left(A_{3}\right),\left(A_{4}\right)$ and $\left(A_{5}^{\prime}\right)$ the closed-loop system (2)-(10) is uniformly exponentially convergent to $B\left(r_{\varepsilon}\right)$ with rate $\alpha$ and region of attraction $R$ where

$$
R=\left\{x \in \mathbb{R}^{n} \mid V(x) \leq \frac{\left(1-k_{2}\right)^{2} \tilde{\rho}^{2} \lambda_{1}}{M^{2} \lambda_{3}^{2}}\right\} .
$$

Proof. Proceeding as in the proof of Theorem 3, we note that, on the one hand, if

$$
\left\|L_{g} V(x)\right\| \leq\left(1-k_{2}\right) \tilde{\rho}
$$

we have

$$
\bar{u}(x)=u(x) .
$$

On the other hand, using assumption $\left(A_{5}^{\prime}\right)$ and equations (6) and (7) we have

$$
\begin{aligned}
\left\|L_{g} V(x)\right\|^{2} & \leq\|D V(x)\|^{2}\|g(x)\|^{2} \\
& \leq \lambda_{3}^{2} M^{2}\|x\|^{2} \\
& \leq \frac{\lambda_{3}^{2} M^{2}}{\lambda_{1}} V(x) .
\end{aligned}
$$


Whenever

$$
V(x) \leq \frac{\left(1-k_{2}\right)^{2} \tilde{\rho}^{2} \lambda_{1}}{M^{2} \lambda_{3}^{2}}
$$

we have

$$
\left\|L_{g} V(x)\right\| \leq\left(1-k_{2}\right) \tilde{\rho} .
$$

And so we can deduce the result by applying Theorem 1, with

$$
V_{1}=\frac{k_{1} \varepsilon}{\alpha}
$$

and

$$
V_{2}=\frac{\left(1-k_{2}\right)^{2} \tilde{\rho}^{2} \lambda_{1}}{M^{2} \lambda_{3}^{2}}
$$

\subsection{Illustrative example.}

Consider the following example:

$$
\begin{aligned}
& \dot{x}_{1}=x_{1}^{2} x_{2}+u+q(t) \\
& \dot{x}_{2}=-x_{1}^{3}+x_{1} \sqrt{1+\sin ^{2} x_{2}}+(u+q(t)) \sqrt{1+\sin ^{2} x_{2}}
\end{aligned}
$$

where $x=\left(x_{1}, x_{2}\right)^{T} \in \mathbb{R}^{2}$ and $q$ stands for an unknown bounded function, that is, there exists $q_{0}>0$ such that $|q(t)| \leq q_{0}, \forall t \geq 0$. It is easy to see that system (11) is under form (2) with

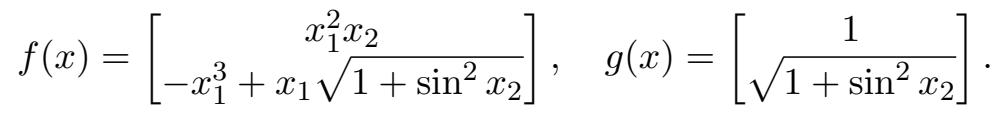

Suppose that the control $u$ is subject to the constraint (4) with $q_{0}<\bar{\rho}$. Therefore assumptions $\left(A_{1}\right),\left(A_{2}\right)$ and $\left(A_{3}\right)$ are satisfied. Let $\alpha=1 / 2$ and $V(x)=(1 / 2) x_{1}^{2}+(1 / 2) x_{2}^{2}$.

$$
\begin{aligned}
& L_{f}(x)=x_{1} x_{2} \sqrt{1+\sin ^{2} x_{2}} \\
& L_{g}(x)=x_{1}+x_{2} \sqrt{1+\sin ^{2} x_{2}} .
\end{aligned}
$$

So

$$
\begin{aligned}
2 \alpha V(x)+L_{f} V(x)-\left(L_{g} V(x)\right)^{2}= & -\frac{1}{2}\left(x_{1}+x_{2} \sqrt{1+\sin ^{2} x_{2}}\right)^{2} \\
& -\frac{1}{2} x_{2} \sin ^{2} x_{2} \\
\leq & 0 .
\end{aligned}
$$

Hence, $V$ is a suitable Lyapunov function which satisfies assumption $\left(A_{4}\right)$ with $\lambda_{1}=\lambda_{2}=1 / 2$ and $\lambda_{3}=1$. Moreover, $\|g(x)\|=\sqrt{2+\sin ^{2} x_{2}} \leq \sqrt{3}$, 
thus, assumption $\left(A_{5}^{\prime}\right)$ is assured with $M=\sqrt{3}$. We can now use Theorem 4 to state that a controller given by (10) with

$$
\varepsilon<\frac{\left(\bar{\rho}-q_{0}\right)^{2}}{12 q_{0}}
$$

yields exponential convergence to $B\left(r_{\varepsilon}\right)$.

Conclusion. Throughout this paper, we have proposed continuous state feedback controllers for a class of uncertain systems that assure global exponential convergence of the solutions towards a neighborhood of the origin. By saturating these states feedback functions outside a compact region, we get bounded state feedback controllers and we show that there is a region of attraction from which solutions of the closed-loop system are uniformly exponentially convergent towards an arbitrary small neighborhood of the origin.

\section{References}

[1] Benabdallah, A., Hammami, M. A., On the output feedback stability for non-linear uncertain control systems, Internat. J. Control 74(6) (2001), 547-551.

[2] Corless, M., Guaranteed rates of exponential convergence for uncertain systems, J. Optim. Theory Appl. 64 (1990), 481-494.

[3] Corless, M., Leitmann, G., Continuous state feedback guaranteeing uniform ultimate boundedness for uncertain dynamic systems, IEEE Trans. Automat. Control 26(5) (1981), 1139-1143.

[4] Corless, M., Leitmann, G., Bounded controllers for robust exponential convergence, J. Optim. Theory Appl. 76(1) (1993), 1-12.

[5] Dawson, D. M., Qu, Z., Carroll, J. C., On the state observation and output feedback problems for nonlinear uncertain dynamic systems, Systems Control Lett. 18 (1992), 217-222.

[6] Gutman, P. O., Hagandar, P., A new design of constrained controllers for linear systems, IEEE Trans. Automat. Control 30 (1985), 22-33.

[7] Hached, M., Madani-Esfahani, S. M., Żak, S. H., On the stability and estimation of ultimate boundedness of nonlinear/uncertain dynamic systems with bounded controllers, Proc. of the American Control Conference (San Diego, CA, 1990) 2 (1990), 1180-1185.

[8] Isidori, A., Nonlinear Control Systems, 3rd edition, Comm. Control Engrg. Ser., Springer-Verlag, Berlin, 1995.

[9] Lewis, F. L., Syrmos, V. L. Optimal Control, Wiley, New York, 1995.

[10] Madani-Esfahani, S. M., Hui, S., Żak, S. H., On the estimation of sliding domains and stability regions of variable structure control systems with bounded controllers, Proc. of the 26th Allerton Conference on Communication, Control and Computing (Monticello, IL, 1988), 518-525.

[11] Madani-Esfahani, S. M., Żak, S. H., Variable structure control of dynamical systems with bounded controllers, Proc. of the American Control Conference (Minneapolis, MN, 1987) 1 (1987), 90-95. 
[12] Sepulchre, R., Janković, M., Kokotović, P., Constractive Nonlinear Control, SpringerVerlag, Berlin-Heidelberg-New York, 1997.

[13] Soldatos, A. G., Corless, M., Stabilizing uncertain systems with bounded control, Dynam. Control 1 (1991), 227-238.

[14] Walcott, B. L., Żak, S. H., State observation of nonlinear uncertain dynamical systems, IEEE Trans. Automat. Control 32(2) (1987), 166-170.

[15] Wu, H., Mizukami, K., Exponential stapbility of a class of nonlinear dynamical systems with uncertainties, Systems Control Lett. 21 (1993), 307-313.

Amel Benabdallah

Mohamed Ali Hammami

FACUlty of Sciences of SfaX

Faculty of Sciences of Sfax

Department of Mathematics

Department of Mathematics

Route Soukra Km 4, B.P. 1171

Route Soukra Km 4, B.P. 1171

3000 SFAX, TUNiSia

3000 Sfax, Tunisia

E-MAil: Amel.Benabdallah@Fss.rnu.Tn

E-MAIL:MOHAMED.HAMMAMI@FSS.RNU.TN 